\title{
Quality control in cloth production: a new system for real-time defect detection
}

\author{
Antonio Baldassarre ; Maurizio De Lucia ; Francesca Rossi ; Massimiliano Vannucci
}

http://proceedings.spiedigitallibrary.org/proceeding. aspx? articleid=927031

SPIE 4191, Sensors and Controls for Intelligent Manufacturing, 45 (November 22, 2000);

doi:10.1117/12.417246

\section{ABTRACT}

Real time defect detection on fine cloth is an urgent problem to solve: detecting a long and serious defect on a roll, as soon as it is produced, can reduce damages to the roll, and the consequent decrement of price. The paper describes the work performed at the Department of Energy Engineering 'Sergio Stecco' of the University of Florence, in collaboration with well-known high quality wool cloth manufacturers (Marzotto) and machine builders (Sulzer, Benninger). The main goal has been to obtain a new and innovative production line, endowed with a system (based on image processing techniques) for detecting defects in real- time and thus for controlling the production process. The system is based on image processing techniques with a special attention to the real-time constraints. An architecture separating an on-line defect detection and an off-line classification has been proposed. An intelligent optical head, assembled on the loom, acquires images and detects the defects in real-time. A server has the offline task to classify each defect detected by the head. The system has been tested on a real loom, with good results in terms of reliability, false alarms and stability. 